\title{
A possible predictive marker of progression for hepatocellular carcinoma
}

\author{
MICHELE DI STASIO $^{1 *}$, MARIA GRAZIA VOLPE ${ }^{*}$, GIOVANNI COLONNA $^{2}$, MELISSA NAZZARO $^{1}$, \\ MIRIAM POLIMENO $^{3}$, STEFANIA SCALA ${ }^{3}$, GIUSEPPE CASTELLO ${ }^{4}$ and SUSAN COSTANTINI ${ }^{4}$ \\ ${ }^{1}$ Institute of Food Sciences, CNR, Avellino; ${ }^{2}$ Department of Biochemistry and Biophysics and Interdepartmental Research \\ Center for Computational and Biotechnological Sciences (CRISCEB), Second University of Naples, Naples; \\ ${ }^{3}$ 'G. Pascale Foundation' National Cancer Institute, Naples; ${ }^{4}$ G G. Pascale Foundation’ National Cancer Institute, \\ Cancer Research Center, Mercogliano, Avellino, Italy
}

Received March 29, 2011; Accepted July 18, 2011

DOI: $10.3892 / \mathrm{ol} .2011 .378$

\begin{abstract}
The correlation between decreased levels of selenium and increased DNA damage and oxidative stress shows the significance of this trace element. A number of studies have provided evidence for lower serum, plasma and tissue levels of selenium in patients with various diseases and types of cancer. In this study, liver selenium concentrations were measured in tissue samples of patients with hepatocellular carcinoma (HCC) by atomic absorption spectrometry. The results showed that the selenium concentrations decreased when the malignant grade increased. Furthermore, a significant correlation was found between selenium levels and human selenium binding protein-1 (SELENBP1) down-regulation in the liver. Therefore, we suggest that the evaluation of selenium and SELENBP1 concentrations can be used for improving the prognosis of HCC.
\end{abstract}

\section{Introduction}

Selenium is a trace element for which no direct sign of its being essential in human nutrition was found until 1979. In the same year, the existence of a correlation between the low concentration of selenium in the geographical area of Keshan in China and the pathology, known as Keshan disease, was identified by a research group in this country (1). Selenium is stored in human tissues in varying amounts: $30 \%$ of tissue selenium is in the liver, $15 \%$ in the kidney, $30 \%$ in muscle, $10 \%$ in the plasma and the remaining $15 \%$ throughout the other organs. Selenium is found in trace quantities in a number of dietary agents such as dairy products, meat products,

Correspondence to: Dr Susan Costantini, INT Pascale - CROM, via Ammiraglio Bianco, 83013 Mercogliano, Avellino, Italy E-mail: susan.costantini@unina2.it

*Contributed equally

Key words: selenium, selenium binding protein-1, hepatocarcinoma prognosis, atomic absorption spectrometry, HCC marker poultry, fish, fruits, vegetables and cereals, rendering it part of the food chain.

Selenium is considered to be a significant antioxidant, a precursor of the antioxidant enzyme known as 'glutathione peroxidase' (GPX), which protects cells from free radical damage in a number of neuronal and neuromuscular disorders, such as stroke and cerebrovascular disease, Alzheimer's disease, Parkinson's disease, familial amyotrophic lateral sclerosis and Duchenne muscular dystrophy. The proposed mechanisms mainly invoke the function of GPX as well as that of selenoprotein P (2). The evidence linking a lack of selenium with cancer is found in epidemiological and clinical studies. Low dietary selenium levels became an accurate way of predicting future cancer rates. A previous study showed that selenium supplementation led to a $50 \%$ reduction in cancer mortality (3). In general, the mean content of selenium in serum from patients with various types of cancer was lower than that of the control groups. Therefore, the correlation between decreased levels of selenium and increased DNA damage and increased oxidative stress further indicates the significance of this trace element (4).

A number of studies, including geographic, pre-clinical animal and prospective, as well as intervention studies, have shown selenium to be involved in a number of types of gastrointestinal cancer, such as esophageal, colorectal and prostate, as well as liver cancer (5-6), and have suggested its putative role in cancer, as well as progression, and, subsequently, metastasis prevention (7). A significant positive correlation between plasma selenium levels and liver cancer was also found. A number of epidemiological studies performed on humans showed significantly lower serum, plasma and liver selenium levels in patients with liver diseases, such as chronic hepatitis and cirrhosis, and different grades of hepatocellular injury, than those measured in the healthy control groups (8-13). Hepatocellular carcinoma (HCC) develops in the liver with severe impairment of cellular antioxidant systems, since, in patients with liver metastases from various types of cancer, cellular scavenger enzymes exhibited normal activities, despite low selenium concentrations (14).

In particular, HCC is a major health problem worldwide, being the fifth most common malignancy in males and 
Table I. Clinicopathological characteristics of HCC patients subdivided according to grading. ${ }^{a}$

\begin{tabular}{|c|c|c|c|c|c|c|c|c|c|}
\hline \multirow[t]{2}{*}{ Grading } & \multicolumn{2}{|c|}{ Age } & \multicolumn{2}{|c|}{ Gender } & \multicolumn{3}{|c|}{ Tumor invasion } & \multicolumn{2}{|c|}{ Tumor size $(\mathrm{cm})$} \\
\hline & $>70$ & $<70$ & M & $\mathrm{F}$ & $\mathrm{T} 1$ & $\mathrm{~T} 2$ & $\mathrm{~T} 3$ & $<4$ & $>4$ \\
\hline I & 1 & 4 & 4 & 1 & 3 & 2 & 0 & 2 & 3 \\
\hline II & 7 & 2 & 7 & 2 & 1 & 3 & 5 & 3 & 6 \\
\hline III & 3 & 3 & 4 & 2 & 2 & 2 & 2 & 3 & 3 \\
\hline
\end{tabular}

${ }^{a}$ The number of patients to which each parameter refers is reported.

the eighth in females and the third most common cause of cancer-related mortality in the world. The incidence of HCC is on the increase, with marked variations among geographic regions and racial and ethnic groups, relative to the exposure to documented environmental risk factors (15). In particular, Southern Italy has the highest rates of HCC in Europe (16). Previously, we evaluated the expression of the human selenium binding protein-1 (SELENBP1) in tissue samples of HCC patients. SELENBP1 is a protein that incorporates exogenously administered radioactive (75Se) sodium selenite in the liver in vivo (17-18). These studies provide evidence that this protein is down-regulated in the liver tissue of HCC patients and that its gradual loss is associated with an increased malignant grade (19).

In the present study, liver selenium levels in tissue samples of Italian HCC patients were measured by atomic absorption spectrometry in order to investigate whether there was a correlation between selenium and SELENBP1 concentrations. To the best of our knowledge, this is the first study that has examined this type of correlation in human liver tissue.

\section{Materials and methods}

Tissue sample. Paraffin-embedded specimens of liver tissue from $20 \mathrm{HCC}$ patients (5 females, 15 males) were analyzed. The clinicopathological characteristics of these patients are shown in Table I. The study included 5 HCC patients with grade I tumors, 9 HCC patients with grade II and $6 \mathrm{HCC}$ patients with grade III tumors. These patients had underlying hepatitis $\mathrm{C}$ virus (HCV)-related cirrhosis and their age ranged between 57 and 82 years. All 20 patients in this study provided informed consent. Liver tissue samples were obtained from patients recruited as part of an ongoing study to investigate associations between cytokine dynamics and liver injury progression. Following standard diagnostic procedures, biopsies were obtained from the liver and were collected for research purposes.

Atomic absorption spectrometer studies. Nitric acid, 65\%; xylene anhydrous, 99\%; formaldehyde and ultra pure water were from Sigma-Aldrich (Steinheim, Germany). Hydrogen peroxide, $30 \%$ and selenium standard $1000 \mathrm{ppm}$ were from Carlo Erba Reagents (Milan, Italy). Each liver tissue was fixed in formaldehyde and embedded in paraffin as part of routine histological processing. For this study, each paraffin-embedded tissue was dissected from the paraffin block to obtain $20-\mu \mathrm{m}$ slices. The preparation of samples was performed according to a previous study (20). The wax was removed by washing $(2 \times 30 \mathrm{~min})$ in xylene. The deparaffinized specimens were dried at $80^{\circ} \mathrm{C}$ for $24 \mathrm{~h}$ and digested with a mixture of $\mathrm{HNO}_{3}$ and $\mathrm{H}_{2} \mathrm{O}_{2}$ at a ratio of $1: 2(\mathrm{v} / \mathrm{v})$ at a temperature of $100^{\circ} \mathrm{C}$ in a bath for $15-20 \mathrm{~min}$.

The selenium concentration was determined by graphite furnace atomic absorption spectroscopy on a Varian SpectrAA 200 (Victoria, Australia) spectrometer with Zeeman background correction. The quantitative determinations were carried out by a calibration curve using selenium standard solutions (5-20 ppb). Digested samples were diluted with ultra pure water to yield the selenium concentration within the calibration range.

The furnace settings were as follows: for drying, ramp to $85^{\circ} \mathrm{C}(5 \mathrm{sec})$, ramp to $95^{\circ} \mathrm{C}(40 \mathrm{sec})$, and ramp to $120^{\circ} \mathrm{C}$ $(10 \mathrm{sec})$; for ashing, ramp to $1000^{\circ} \mathrm{C}(5 \mathrm{sec})$ and hold at $1000^{\circ} \mathrm{C}$ ( $3 \mathrm{sec})$; for atomization, ramp to $2600^{\circ} \mathrm{C}(0.8 \mathrm{sec}$, read signal), hold at $2600^{\circ} \mathrm{C}\left(2 \mathrm{sec}\right.$, read signal), and hold at $2600^{\circ} \mathrm{C}(2 \mathrm{sec}$, tube clean). The absorbance was determined at $196.0 \mathrm{~nm}$, and the slit was $1.0 \mathrm{~nm}$.

Data analysis. Selenium concentrations (expressed in $\mu \mathrm{g} / \mathrm{g}$ ) are reported as the arithmetic means \pm standard deviation for each group of patients. The non-parametric Mann-Whitney U test was used to evaluate differences between selenium concentrations from patients with grades I-III HCC. The test was capable of distinguishing values where $p<0.05$ with one asterisk ( $\left.{ }^{*}\right)$, with two asterisks $\left(^{* *}\right)$ where values were $\mathrm{p}<0.01$ and with three asterisks $\left({ }^{* * * *}\right)$ where values were $p<0.0001$. The correlations between the selenium levels and clinical data or immunohistochemical scores related to SELENBP1 (19) were determined using the Pearson's correlation coefficient. $\mathrm{P}<0.05$ was considered to be statistically significant. The statistical program Prism 4 (GraphPad Software, San Diego, CA, USA) was employed.

The relationship degrees between selenium levels and patients were studied by means of the hierarchical agglomerative clustering method. Cluster analyses were performed using the STATISTICA 8.0 statistical package (Statsoft Inc., Tulsa, OK, USA). In particular, the patients were assigned to clusters step-by-step. Following each step, clusters were joined to form new clusters. The course of this process of increasing the degree of clustering was exhibited in a dendrogram. At any distance from the start of the clustering process, the number of clusters formed at that point are shown in the 


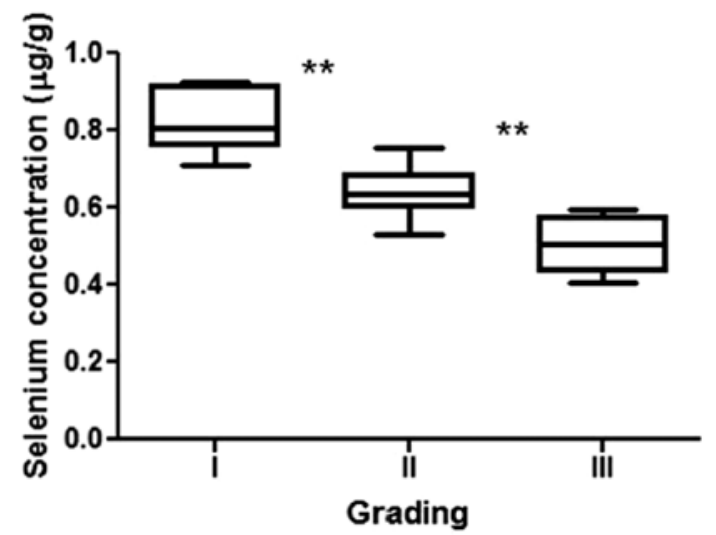

Figure 1. Selenium concentrations ( $\mu \mathrm{g} / \mathrm{g}$ of liver) in HCC patients with grades I-III were plotted with box-and-whisker graphs. The boxes extend from the 25 th to the 75 th percentile, and the line in the middle is the median. The error bars extend down to the lowest value and up to the highest. ${ }^{* *} \mathrm{P}<0.01$.

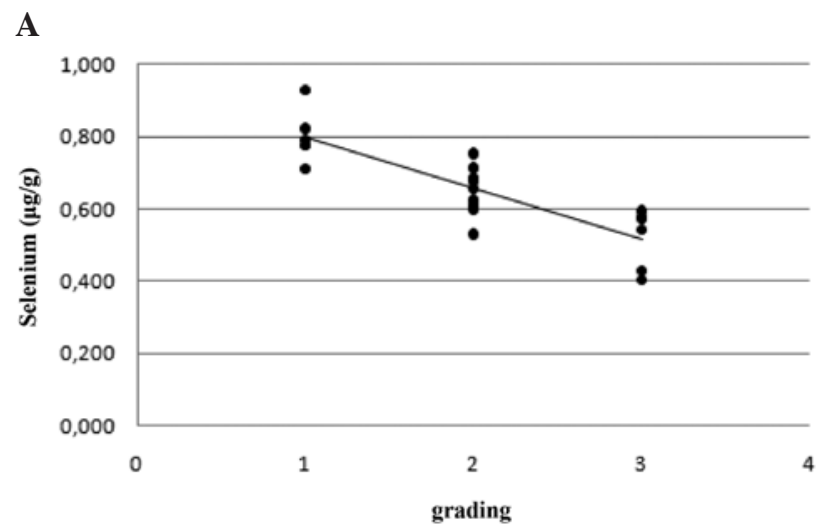

B

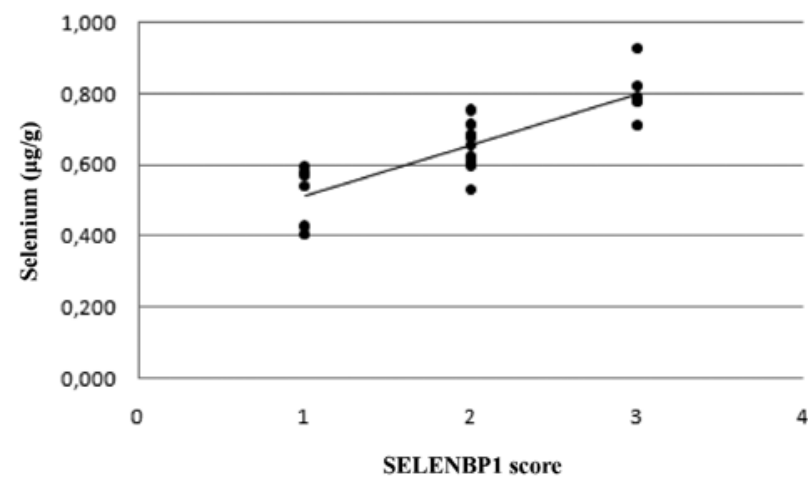

Figure 3. (A) Correlation between tissue selenium concentrations $(\mu \mathrm{g} / \mathrm{g})$ and grading or (B) immunohistochemical scores of SELENBP1.

graph (Fig. 1). The basis for a cluster analysis was formed by calculating the degree of similarity among the relevant variables of the different patients to be clustered. In this study, the Euclidean distance between patients was selected as the measure of similarity (21-22). Although this study included a relatively small number of patients, the approach of selecting

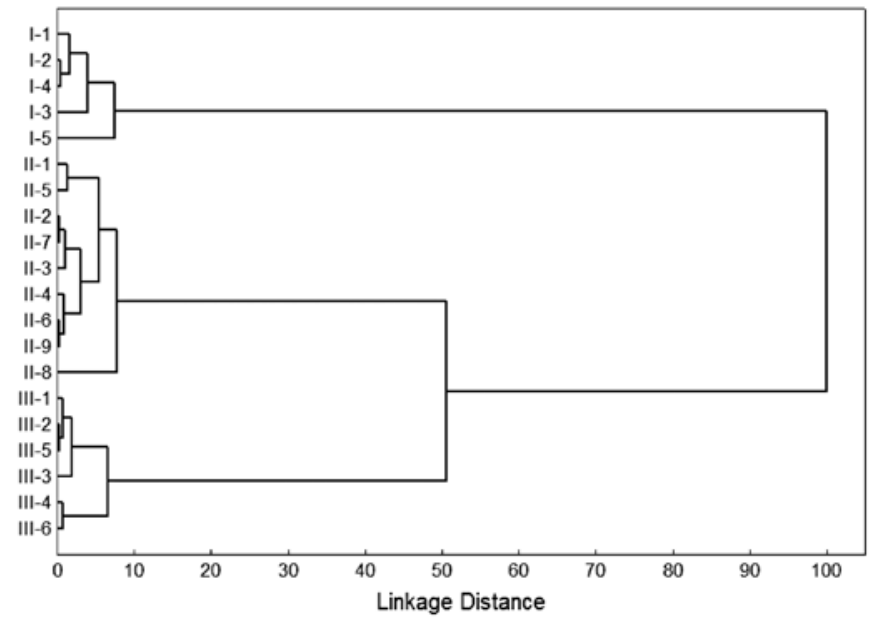

Figure 2. Dendrogram of the cluster analysis. At a distance level of 10, three clusters are distinguished; the horizontal axis is scaled in terms of percentages.

these patients for high, medium and low malignant grade is a way in which to increase the statistical power.

\section{Results}

In a recent study, we found that the human SELENBP1 is also down-regulated in HCC (19). The present study correlates the amount of selenium in HCC patients with the liver content of this protein. The concentration of selenium in HCC patients subdivided into three groups based on malignant grades (I-III) has been evaluated by atomic absorption spectrometry (Fig. 1). The mean concentrations found in the liver were $0.823 \pm 0.083 \mu \mathrm{g} / \mathrm{g}$ of selenium for $\mathrm{HCC}$ patients with grade I, $0.638 \pm 0.066 \mu \mathrm{g} / \mathrm{g}$ for those with grade II and $0.505 \pm 0.076 \mu \mathrm{g} / \mathrm{g}$ for those with grade III. These values are lower than those found in the livers of healthy individuals $(1.9 \pm 0.6 \mu \mathrm{g} / \mathrm{g})(23)$. The results are also in agreement with scattered data reported in other studies (8-13).

We compared the selenium levels in three groups using the Mann-Whitney U test. The differences between the patients with grade I and II or with grade II and III are statistically significant with $\mathrm{p}=0.0017$ and $\mathrm{p}=0.0018$, respectively. This finding is also confirmed by the hierarchical clustering analysis of patients based on the selenium concentrations. The analysis provides evidence that the patients were placed into three groups, corresponding to the three different malignant grades (Fig. 2). Moreover, the data highlight that selenium concentrations decrease when the malignant grade increases with a correlation coefficient equal to -0.86 (Fig. 3A), whereas no correlation has been observed between selenium levels and patient characteristics such as age/gender, tumor invasion and tumor size. Finally, we have correlated the selenium concentrations of patients with grades I-III HCC and the expression of SELENBP1, which was recently evaluated in the same patients, by immunohistochemical studies (19). The rationale of this evaluation is based on the fact that this protein incorporates exogenously administered sodium selenite in the liver. Our studies provided evidence that SELENBP1 was also down-regulated in the liver tissues of HCC patients and its gradual loss was associated with an increased malignant 
grade (19). As reported in Fig. 3B, a significant correlation between SELENBP1 expression and selenium levels with a correlation coefficient equal to 0.83 was detected. Thus, a decrease in selenium concentration in the liver corresponds to lower values of SELENBP1. This is a notable result since we have shown a clear correlation with SELENBP1 in liver cancer, while data published in a recent study (24) report no correlation between serum or prostate tissue selenium and GPX activity in prostate cancer patients. It is noteworthy that prostate cancer also shows SELENBP1 down-regulation (25). Therefore, in general, these data appear to suggest that the selenoenzymes containing endogenous selenium as selenocysteine are not involved in those mechanisms related to the low selenium levels in HCC tissues.

\section{Discussion}

Therapy for HCC remains poor; therefore, it is essential to identify novel HCC markers for early detection of this disease, and tumor-specific proteins as potential therapeutic targets. Despite the various studies conducted to understand the clinicopathological characteristics of the disease and to improve patient treatment, conventional clinicopathological characteristics have a limited predictive capacity. In 1960, it was suggested that $\alpha$ fetoprotein (AFP) may be a useful tumor marker for HCC (26). Although AFP is widely used as a marker, its drawbacks include a limited sensitivity and the fact that AFP is not specific to HCC (27). Thus, the diagnosis of HCC cannot depend mainly on the measurement of AFP. Consequently, molecular biomarkers associated with HCC should be identified. Different proteomics have been applied to the study of HCC, resulting in panels of altered proteins that are proposed as markers with key roles in the progression of the disease in certain cases (28-35). However, the protein repertoires reported in these studies are barely coincident and, consequently, their value as biomarkers for HCC is limited. At present, only five biomarkers for HCC are available for clinical use, from which only AFP partially fulfills the requirements of an ideal tumor marker (36).

Selenium is capable of being tightly bound by proteins, known as selenium-binding proteins, to distinguish them from real selenoproteins (37). The exact role of selenium-binding proteins is unknown, although certain evidence suggests that these proteins are involved in intra-Golgi protein transport. A homolog in Lotus japonicus has been found to be involved in the modulation of the oxidation/reduction status of target proteins in vesicular Golgi transport (38-39). Moreover, selenium has been found to be involved in the protection from ROS-induced cell damage, which is evident in a number of diseases, and its serum and tissue levels have been found to be significantly lower in patients with certain types of cancer in respect to healthy controls (5-6). Therefore, a functional correlation may be suspected between the antique protein of Lotus and that in humans. The Golgi apparatus has the crucial cellular function of protein release following protein biosynthesis on ribosomes; therefore, a functional protection against oxidative phenomena always present in the eukaryotic cell may be necessary.

In this study, we have evaluated liver selenium levels in tissue samples of HCC patients subdivided on the basis of grading by atomic absorption spectrometry. The mean selenium concentrations in the liver were lower in patients with the highest malignant grade in the following order: grade $\mathrm{I}>$ grade II $>$ grade III. Moreover, these values correlated with the immunohistochemical scores related to SELENBP1 evaluated in a recent study (19). Therefore, the results suggest that i) the evaluation of selenium and SELENBP1 concentrations are particularly useful for prognosis improvement when clinical considerations suggest a hepatic biopsy in cirrhotic patients, and ii) the intake of selenium and its derivatives in cirrhotic patients should be considered as potential drugs against HCC, which is in agreement with a number of studies on human hepatoma cells and on animal models of liver cancer that showed the cytotoxic and antioxidant effects of selenium (40). To the best of our knowledge, no study has investigated the correlation between selenium and SELENBP1 concentrations in the liver tissue of patients affected by HCC, being a potentially significant target tissue for a chemopreventive effect of selenium. Although there is no other human tissue study, a number of studies investigated circulating concentrations of selenium, which were used as a surrogate for tissue measurements. Our findings are specific for HCC, however we suggest that this correlation should be confirmed for other types of cancer in which down-regulation of SELENBP1 has been found.

\section{References}

1. Keshan Disease Research Group: Epidemiologic studies on the ethiologic relationship of selenium and Keshan disease. Chi Med 92: 477-482, 1979.

2. Sagara Y, Tan S, Maher P and Schubert D: Mechanisms of resistance to oxidative stress in Alzheimer's disease brain. Neurotoxicology 19: 339-345, 1998.

3. Clark LC, Combs GF Jr, Turnbull BW, et al: Effects of Selenium supplementation for cancer prevention in patients with carcinoma of the skin. JAMA 276: 1957-63, 1996.

4. Rayman MP: Selenium in cancer prevention: a review of the evidence and mechanism of action. Proc Nutr Soc 64: 527-542, 2005.

5. Peters U and Takata Y: Selenium and the prevention of prostate and colorectal cancer. Mol Nutr Food Res 52: 1261-1262, 2008.

6. Trottier G, Boström PJ, Lawrentschuk $\mathrm{N}$ and Fleshner NE: Nutraceuticals and prostate cancer prevention: a current review. Nat Rev Urol 7: 21-30, 2009.

7. Yoo MH, Xu XM, Carlson BA, Patterson VN, Gladyshev AD and Hatfield DL: Targeting thioredoxin reductase 1 reduction in cancer cells inhibits self-sufficient growth and DNA replication. PLoS One 2: 1112, 2007.

8. Lecomte E, Herberth B, Pirrollet P, et al: Effect of alcohol comsumption on blood antioxidant nutrients and oxidative stress indicators. Am J Clin Nutr 60: 255-261, 1994.

9. Loguercio C, De Girolamo V, Federico A, et al: Trace elements and chronic liver diseases. J Trace Elem Med Biol 11: 158-161, 1997.

10. Guarini P, Stanzial AM, Olivieri O, et al: Erythrocyte membrane lipids and serum selenium in post-viral and alcoholic cirrhosis. Clin Chim Acta 270: 139-150, 1998.

11. Navarro-Alarcòn $M$ and Lòpez-Martìnez MC: Essentiality of selenium in the human body: relationship with different diseases. Sci Total Environ 249: 347-371, 2000.

12. Navarro-Alarcòn M, Lòpez-G de la Serrana H, Pèrez-Valero V and Lòpez-Martìnez MC: Selenium concentrations in serum of individuals with liver diseases (cirrhosis or hepatitis): relationships with some nutritional and biochemical markers. The Science of the total environment 291: 135-141, 2002.

13. Tashiro H, Kawamoto T, Okubo T and Koide O: Variation in the distribution of trace elements in hepatoma. Biol Trace Element Research 95: 49-63, 2003

14. Casaril M, Corso F, Bassi A, et al: Decreased activity of scavenger enzymes in human hepatocellular carcinoma, but not in liver metastases. International Journal of Clinical and Laboratory Research 24: 94-97, 2006. 
15. Castello G, Scala S, Palmieri G, Curley SA and Izzo F: HCV-related hepatocellular carcinoma: from chronic inflammation to cancer. Clin Immunol 134: 237-50, 2010.

16. Fusco M, Girardi E, Piselli P, et al: Epidemiology of viral hepatitis infections in an area of southern Italy with high incidence rates of liver cancer. Eur J Cancer 44: 847-53, 2008.

17. Bansal MP, Mukhopadhyay T, Scott J, Cook RG, Mukhopadhyay R and Medina D: DNA sequencing of a mouse liver protein that binds selenium: implications for selenium's mechanism of action in cancer prevention. Carcinogenesis 11: 2071-2073, 1990

18. Chang PW, Tsui SK, Liew C, Lee CC, Waye MM and Fung KP Isolation, characterization, and chromosomal mapping of a nove cDNA clone encoding human selenium binding protein. J Cell Biochem 64: 217-224, 1997.

19. Raucci R, Colonna G, Guerriero E, et al: Structural and functional studies of the human selenium binding protein-1 and its involvment in hepatocellular carcinoma. Biochimi et Biophys Acta 1814: 513-522, 2011.

20. Beilby JP, Prins AW and Swanson NR: Determination of hepatic iron concentration in fresh and paraffin-embedded tissue. Clinical Chemistry 45: 573-574, 1999.

21. Everitt BS, Landau S and Leese M: Cluster analysis. New York: Oxford Univ Press 2001.

22. Hartigan JA: Clustering algorithms. New York: John Wiley and Sons Inc., 1975

23. Oldereid NB, Thomassen Y and Purvis K: Selenium in human male reproductive organs. Human Reproduction 13: 2172-2176, 1998.

24. Takata Y, Morris JS, King IB, Kristal AR, Lin DW and Peters U: Correlation between selenium concentrations and glutathione peroxidise activity in serum and human prostate tissue. The Prostate 69: 1635-1642, 2009.

25. Jeong JY, Wang Y and Sytkowski AJ: Human selenium binding protein-1 (hSP56) interacts with VDU1 in a selenium-dependent manner. BBRC 379: 583-588, 2009.

26. Johnson P: Role of $\alpha$-fetoprotein in the diagnosis and management of hepatocellular carcinoma. Gastroenterol and Hepatol 14: S32-S36, 1999.

27. Nguyen M, Garcia R, Simpson P, Wright T and Keeffe B: Racial differences in effectiveness of $\alpha$-fetoprotein for diagnosis of hepatocellular carcinoma in hepatitis $\mathrm{C}$ virus cirrhosis Hepatology 6: 410-417, 2002.

28. Kim W, Oe Lim S, Kim JS, et al: Comparison of proteome between hepatitis B virus- and hepatitis C virus-associated hepatocellular carcinoma. Clin Cancer Res 9: 5493-5500, 2003.
29. Kuramitsu Y and Nakamura K: Current progress in proteomic study of hepatitis C virus-related human hepatocellular carcinoma. Expert Rev Proteomics 2: 589-601, 2005.

30. Li C, Hong Y, Tan YX, et al: Accurate qualitative and quantitative proteomic analysis of clinical hepatocellular carcinoma using laser capture microdissection coupled with isotope-coded affinity tag and two-dimensional liquid chromatography mass spectrometry. Mol Cell Proteomics 3: 399-409, 2004.

31. Liang CR, Leow CK, Neo JC, et al: Proteome analysis of human hepatocellular carcinoma tissues by two-dimensional difference gel electrophoresis and mass spectrometry. Proteomics 5: 2258-2271, 2005.

32. Lim SO, Park SJ, Kim W, et al: Proteome analysis of hepatocellular carcinoma. BBRC 291: 1031-1037, 2002.

33. Blanc JF, Lalanne C, Plomion C, et al: Proteomic analysis of differentially expressed proteins in hepatocellular carcinoma developed in patients with chronic viral hepatitis C. J Proteomics 5: 3778-3789, 2005.

34. Kim J, Kim SH, Lee SU, et al: Proteome analysis of human liver tumor tissue by two-dimensional gel electrophoresis and matrix assisted laser desorption/ionization-mass spectrometry for identification of disease-related proteins. Electrophoresis 23: 4142-4156, 2002

35. Paradis V, Degos F, Dargere D, et al: Identification of a new marker of hepatocellular carcinoma by serum protein profiling of patients with chronic liver diseases. Hepatology 41: 40-47, 2005.

36. Santamaría E, Muñoz J, Fernández-Irigoyen J, et al: Molecular profiling of hepatocellular carcinoma in mice with a chronic deficiency of hepatic S-adenosylmethionine: relevance in human liver diseases. J Proteome Res 5: 944-953, 2006.

37. Kryukov GV, Castellano S, Novoselov SV, et al: Characterization of mammalian selenoproteomes. Science 300: 1439-1443, 2003.

38. Porat A, Sagiv Y and Elazar Z: A 56-kDa selenium-binding protein participates in intra-Golgi protein transport. J Biol Chem 275: 14457-65, 2000.

39. Flemetakis E, Agalou A, Kavroulakis N, et al: Lotus japonicus gene Ljsbp is highly conserved among plants and animals and encodes a homologue to the mammalian selenium-binding proteins. Mol Plant Microbe Interact 15: 313-22, 2002.

40. Darvesh AS and Bishayee A: Selenium in the prevention and treatment of hepatocellular carcinoma. Anti Canc Agents Med Chem 10: 338-345, 2010. 